\title{
Research on Intelligent Human-Machine Interface of Relay Protection
}

\author{
Can Bao ${ }^{1, a}$, Jianfei Yang and Huaren Wu \\ ${ }^{1}$ School of Electrical and Automation Engineering,Nanjing Normal University,210042 Nanjing, China \\ abcmourn@163.com
}

Keywords: DSP, touch screen, human-machine interface, multilevel menu, relay protection.

Abstract. This paper mainly introduces that intelligent human-machine interaction is realized by using the TI company's DSP chip as the control chip in the embedded system. The design which takes the OCM320240T350--1C touch screen of Jingpeng company as a specific development object uses the DSP chip to control the touch screen to implement the switching display of multilevel menu by using the touch function successfully. The results of the menu operation are accurate and the software program is easy to modify. The universal property of this method is high. The design scheme of this paper will be applied to the human-machine interface of relay protection device of power system. The device can do the transmission experiment, modify the setting value, analyze the fault and so on through the human-machine interface.

\section{Introduction}

In the field of power system relay protection, digital signal processor has been widely used for its high speed processing capacity, rich internal resources, improving cost ratio and many other advantages. The application of digital signal processor is one of the most popular technologies in the field of embedded system development. Human-machine interface is an important part of the power system relay protection device, which is easy to observe the running results or call out the instructions. Human-machine interface is the medium of interaction between operator and machine, it is a trend to develop human-machine interface with touch screen. Human-machine interface based on touch screen combines the buttons and LCD screen, which reduces the volume of human-machine interface device and saves many hardware resources. This paper gives the design scheme that uses the OCM-1C touch screen as the development object and TMS320F28335DSP as the control chip. The design scheme realize the multilevel menu touch operation successfully.

\section{Hardware Design}

This paper use the OCM-1C touch screen of Jinpeng company as an example. The manufacturers have assembled the LCD driver circuit in the touch screen. The touch screen only provides command communication interface for developer to develop. The definitions of each pin are showed in the following Table 1.

Table 1 Pin definition of serial communication interface

\begin{tabular}{cccc}
\hline Number & Name & Type & Illustration \\
\hline 1.2 & VCC & input & Power supply \\
3 & BUSY & output & Busy signal output(Suspended usually) \\
4 & DOUT & output & Transmit pin(TXD),connected to the RXD pin of MCU \\
5.6 & DIN & input & Receive pin(RXD),connected to the TXD pin of MCU \\
7.8 & GND & input & Ground \\
\hline
\end{tabular}

The touch screen communicates with external control chip through RS232 serial communication interface. The serial baud rate is 9600 bits per second. The SCI module of TMS320F28335 supports CPU to communicate with asynchronous peripheral equipment using non-return-to-zero standard format. This paper uses the SCIC module of TMS320F28335 working in half duplex mode. Because the communication interface uses RS232 level, the transmit pin (SCICTXD) and receive pin (SCIRXD) of the SCIC module of TMS320F28335 should connect to the communication port of touch screen through RS232 and TTL/CMOS level conversion circuit. The circuit is shown in Fig.1.SCITXDC and SCIRXDC come from the transmit pin and receive pin of DSP SCIC module. 
The 232_OUT should connect to the DIN pin of touch screen,232_IN to the DOUT,DGND to the GND.

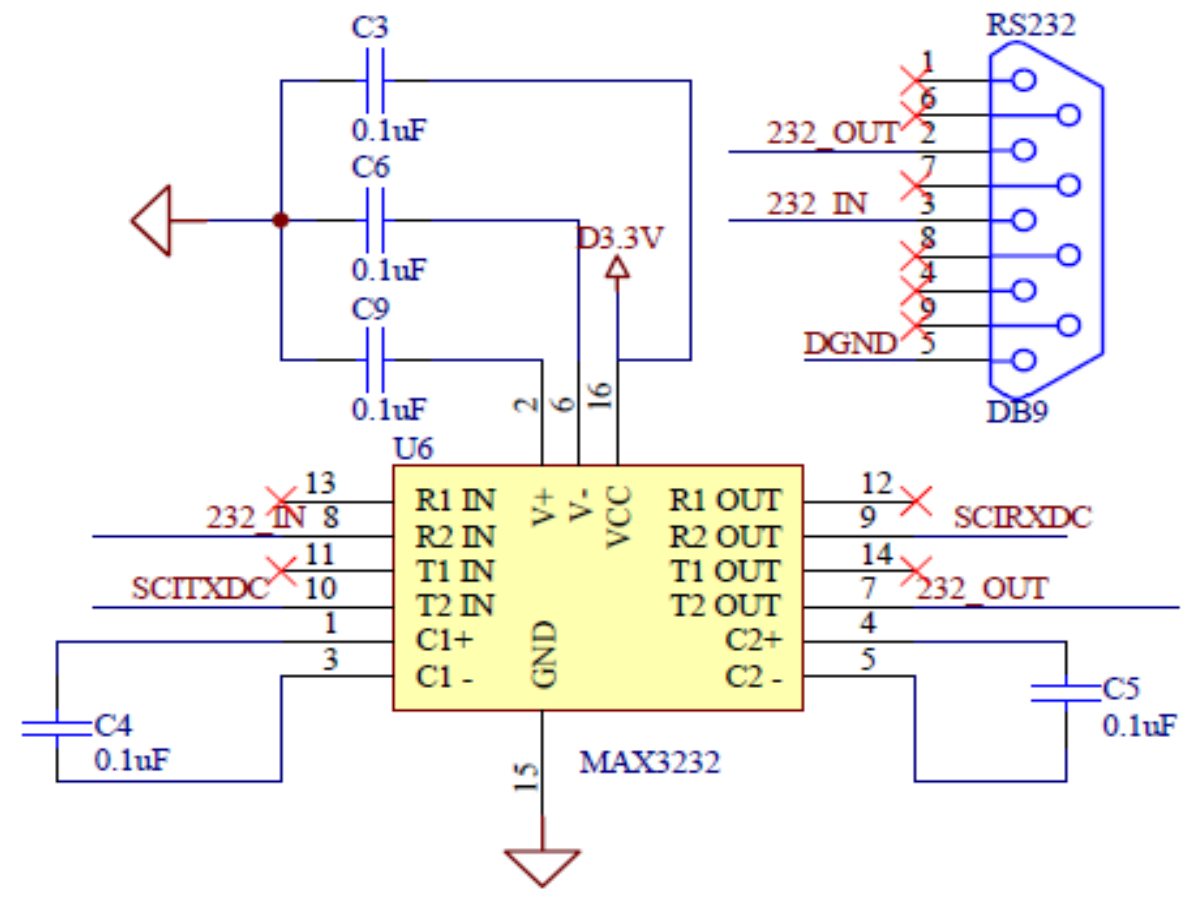

Fig. 1 Level conversion circuit

\section{Software Design}

The manufacturer has assembled the LCD driver circuit in the touch screen. According to the development manual provided by the manufacturer, DSP send specified bytes to the screen through the serial communication interface to control the touch screen display. When the touch screen is pressed, the screen send specified bytes to DSP.DSP can get the coordinates of the touch screen press from these bytes received.

DSP Resource Allocation. DSP writes a byte to the transmit data buffer register(SCITXBUF) to start SCITXINT transmit interrupt. The bytes sent by the touch screen are sent to the SCI receiver shift register (RXSHF), and the bytes enter the receiving data buffer register(SCIRXBUF)to start the SCIRXINT receive interrupt.

In the system initialization function(InitSysCtrl()),the program set CPU clock 150MHZ and enable the SCIC module clock. The transmit interrupt function and receive interrupt function are defined in the program. The address of the interrupt functions should be written into the PIE interrupt vector table. The program is as follows.

PieVectTable.SCIRXINTC=\&SCICRXINTC_ISR;

PieVectTable.SCITXINTC=\&SCICTXINTC_ISR;

In the SCIC module initialization function(InitScic()),Serial communication interface $C$ is set to work in half duplex mode, serial baud rate 9600 bits per second,1 stop bit,8 data bits, no parity. Because the GPIO pin of DSP is multiplexed, the program needs to configure the GPIO63 and GPIO62.These two GPIO pins are selected for receive pin and transmit pin.

Touch Screen Driver Function. The manufacturer has assembled the LCD driver circuit in the touch screen. Chinese characters use 32*32 dot matrix,GB2312 encoding. English character use 16*32 dot matrix, ASCII encoding. The program defines the function named lcdtext3232(int xh,int xl,int yh,int yl,int num,int tab[]) to display Chinese characters and the function named lcdtext1632(int xh,int xl,int yh,int yl,int num,int tab[]) to display English characters. There are 6 arguments passed when the function is called.

Similarly, the program defines the function named clearlcd() to clear the screen, the function named inicolor() to set foreground and background color, the function named touchpanel() to configure touch screen and the function named invertcolor() to invert color. To invert color when the 
screen is pressed, the function exchange the foreground color and background color, then display the same characters. These functions write specified bytes to the transmit data buffer register and wait until the byte sent to the screen one by one. The main function flow chart is shown in Fig. 2 .

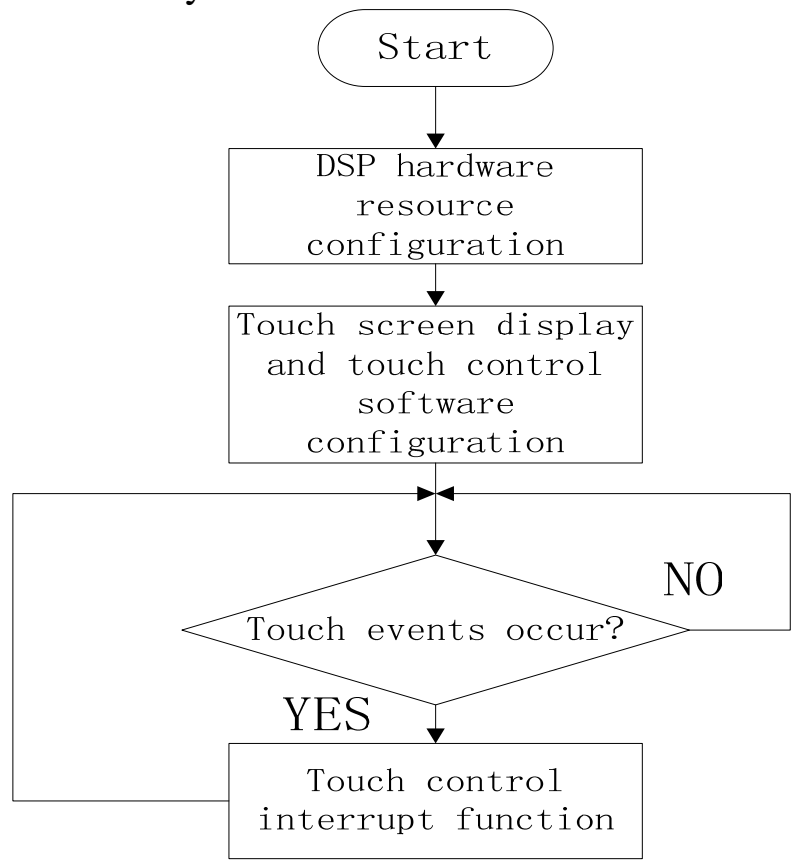

Fig. 2 Main function flow chart

Touch Control Function. The touch screen uploads specified bytes when it is pressed. After DSP receive bytes from touch screen, the program will execute the receive interrupt function. In receive interrupt function, bytes received are stored in array temp[10].According to the event flag byte in the array, the press event flag will be true when touch screen is pressed and press release event flag will be true when the press released. The $\mathrm{X}$ and $\mathrm{Y}$ axis coordinates of touch point can also be get from the array temp[10].The program defines a function named getkeyid(touchx,touchy) that return the key value according to the coordinates.

Implementation of Multilevel Menu Display. When touch control event occurs, DSP determine the new interface according to the current display interface and key value. The program defines two global variables and one function pointer:Current_keyid, Current_menuid, void (*menufunction)(void). Current_keyid indicates the key value that is pressed. Current_menuid indicates the current interface number. Menufunction indicates the sub function that should be called.Current_keyid and Current_menuid determine the sub function that should be called after the key release event is triggered. Current_menuid will be updated in the sub function and Current_keyid come from the function getkeyid(touchx,touchy).

In the program, we define such a structure type containing three elements: interface number, key value and key response function(function pointer).The program is as follows.

typedef struct $\{$ int menuid; int keyid; void (*keyfunction)(); $\}$ menuoperatestruct;

We define a structure array of this type as follows.

menuoperatestruct menuoperate[size $]=\{\{0,6$,lcdmenu1 $\},\{1,0$,lcdmenu3 $\}, \ldots \ldots \ldots . . . .$.$\} ;$

Each structure variable in the array is the logical combination of interface number, the key value in this interface and the sub function which should be called after this key in this interface is pressed. The array contains all of the logical combinations. Current_menuid is updated in these sub functions.

To realize multilevel menu display, the touch control function compares Current_menuid and Current_keyid with menuid and keyid from each element of the array(menuoperate[size]) after getting the Current_keyid. If the program find such an array element, the sub function pointed by function pointer(* keyfunction)in this array element will be called. The touch screen will display a new interface. To add or delete some interfaces, we only need to add or delete corresponding structure elements in the array. The flow chart of receive interrupt function is shown in Fig.3. 


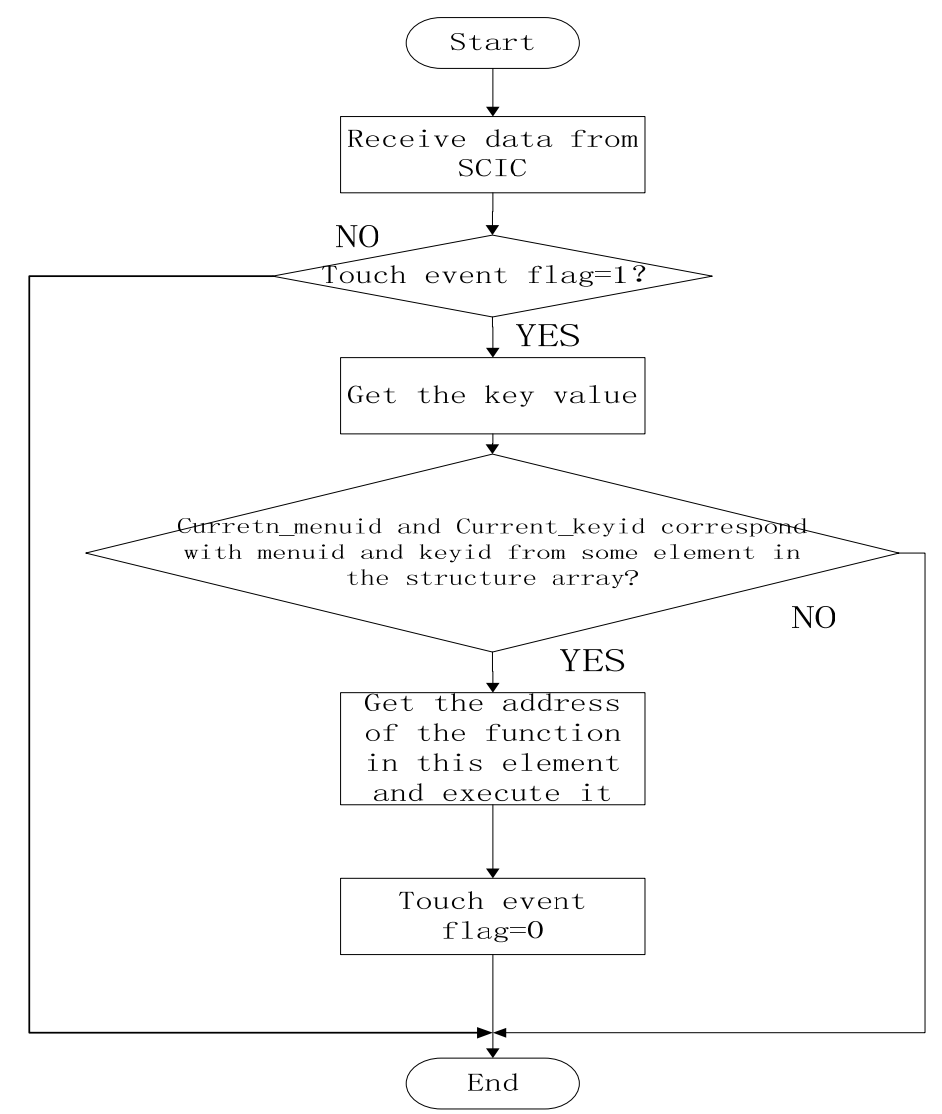

Fig. 3 Receive interrupt function flow chart

\section{Example}

The research in this paper is applied to the human-machine interface of relay protection device of power system. The human-machine interface can achieve many functions of power system relay protection. The examples are shown in Fig.4 and Fig.5.Fig.4 is main menu interface. There are four keys in main menu interface. The screen displays superior menu when key named 'BACK' is pressed. If we press the second key in main menu interface, the screen displays event report interface like Fig.5.If we press the first key in event report interface, touch screen displays trip event interface .

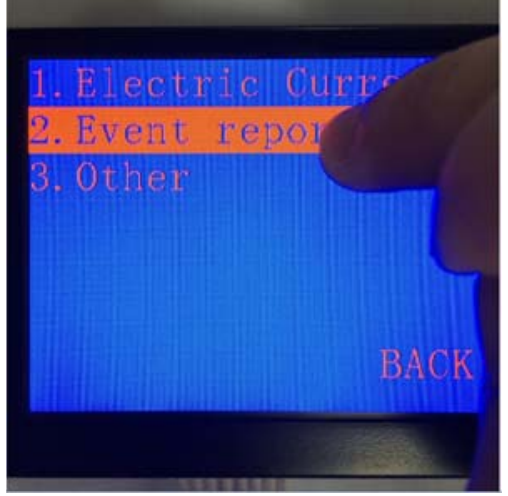

Fig. 4 Main menu interface

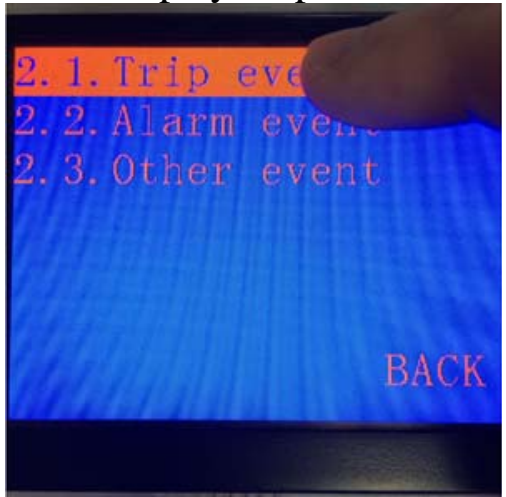

Fig. 5 Event report interface

\section{Conclusions}

Research on intelligent human-machine interface in this paper uses the DSP chip to control the touch screen to implement human-machine interaction successfully. Such a human-machine interface saves the equipment volume and a large amount of hardware resources. This interface makes human machine interaction simple and convenient. In the software design, this paper uses a method of using a structure array to realize multilevel menu display. This method is universal and portable. 


\section{Acknowledgements}

This work was financially supported by the National Natural Science Foundation of China (51177074,51407095) and Jiangsu province natural science foundation (BK20151548).

\section{References}

[1] Xizhen Zheng, Coal Technology, 2011, 30 (2): 190-192.In Chinese

[2] Mingyuan Yang,Ying He, Journal of Liquid Crystals and Display, 2009, 23 (6): 760-764.In Chinese

[3] Hongshe Dang,Yong Yao ,Xinyuan Zhang,Computer Applications and Software, 2013,10:159-161+188.In Chinese

[4] Wei Tang, Fan Xia, Di Sun,Peng Zhang, Automation and Instrumentation, 2012,09:56-60.In Chinese

[5] Xiaohui Song,Hua Ye,Hao Ding, Journal of Southeast University (Natural Science Edition),2007,S1:66-70。In Chinese

[6] Wang X F, Meng G X, Xie W H, et al.. Electric Welding Machine, 2010.

[7] Liu X S, Yang J H.. Microprocessors, 2010. 